P-42 POSITIVE CONTACT IN PALLIATIVE CARE? PILOT STUDY: EFFICACY OF PERSON-CENTRED COMPLEMENTARY THERAPIES IN IMPROVING THE QUALITY OF LIFE OF CARE HOME SERVICE USERS

Louise Graham, Louise Gray. Kilbryde Hospice, East Kilbride, UK

10.1136/bmjspcare-2016-001245.66

Background Following evaluation of our Care@Home project, it was noted that no referrals had been received for care home service users (CHSU). Given current research regarding end-of-life care in care homes, discussions were held with health and social care colleagues. As a result two local care homes agreed to be part of this pilot study.

Aims To identify if an existing service in patients' homes could be transferred to a care home setting, measuring impact on palliative care CHSU displaying physical and/or psychological symptoms of their life-limiting, progressive illness.

Methodology A prospective pilot study of complementary therapy provision, utilising snowball sampling of CHSU $(n=8)$ in collaboration with care home staff, using mix of assessment tools to capture and measure pre/post therapy data.

Results To date, data captured from assessment tools indicates a $100 \%$ reduction in non-verbal signs of distress, and a $77 \%$ reduction in physical changes (arm and hand contractures). Qualitative feedback from service users/family members/care home staff has shown a $100 \%$ improvement in sleep pattern, agitation, anxiety, tension and responsiveness, and a 75\% improvement in diet. In those CHSU where constipation was cited as a symptom, IPOS ${ }^{2}$ results indicate a $100 \%$ reduction in constipation.

However, both the DisDATresults and qualitative feedback show little post-therapy improvement in confusion levels.

Conclusions From results received to date, the authors conclude that it would be beneficial to continue providing complementary therapies to CHSU. Further funding would allow this continuation, as well as study expansion/further research.

The authors' employer funded this study.

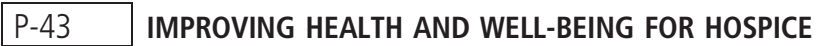 PATIENTS THROUGH SOCIAL AND THERAPEUTIC HORTICULTURE}

Stephen Oxberry, Elspeth McGloughlin, Michael Crowther. Kirkwood Hospice, Huddersfield, UK

\subsection{6/bmjspcare-2016-001245.67}

Grow Group is a hospice-based weekly horticulture group for inand out-patients, set in our Support and Therapy day centre. It was established as a result of an audit which highlighted the need to increase the range of therapeutic activities available to patients, providing services that may be of interest to a wider range of patients than just traditional group activities. Therapeutic horticulture is considered to have benefits to physical, psychological and psychosocial health and well-being.

The aims of the Grow Group were; to improve patients' health and well-being through the use of social and therapeutic horticulture, provide opportunity for engagement in activities meaningful to patients, to provide opportunity for skill maintenance and acquisition, to increase the range of activities available to patients, maintain levels of engagement and enable patients to have an increased internal locus of control.

The weekly sessions are facilitated by an occupational therapist with the assistance of volunteers. Activities and tasks are adapted to meet the needs of patients, and are delivered both inside and outside dependent on weather conditions. The activities enable engagement in meaningful occupations despite physical or cognitive impairment in a physically and socially supportive environment, giving an element of choice and opportunity for decision making, in addition to the using physical, cognitive and social skills.

The Grow Group is in part self-funded due to income generated through plant sales and part in donations. 41 people have attended over the past eight months. Outcomes are measured objectively in conjunction with gaining qualitative feedback from patients and feedback has been excellent.

We have extended our range of therapeutic interventions and offer opportunity for patients to engage in meaningful occupations and recommend social and therapeutic horticulture as a highly effective therapeutic tool which can potentially greatly improve quality of life.

\section{P-44 HORTICULTURAL THERAPY GROUP}

Dawn Harvey. Ashgate Hospice, Chesterfield, UK

\subsection{6/bmjspcare-2016-001245.68}

Background Following a presentation to therapy colleagues on the benefits of horticultural therapy (HT) for clients with a range of health problems it was felt that clients attending our day hospice may benefit from a similar project.

During conversations with day hospice attendees it was found that several clients had voiced their sadness that they were no longer well enough to tend to their own gardens and it was something they dearly missed. Following meetings with our colleagues, the therapy team agreed to pilot a six- month HT group.

Two small successful funding applications allowed us to start in the Spring of 2016.

Aim To provide two sessions per week, alternating days on a fortnightly basis. Each session lasting for approx one hour to include a variety of indoor and outdoor activities and run by an occupational therapist, assisted by our TI and faithful band of volunteers.

Method Participation is voluntary. Numbers vary week on week dependent on the changing nature of their health status. Whilst there is a degree of planning we are guided by what clients and volunteers bring with them and their own ideas thereby ensuring they feel fully included in our overall aim to develop a patio are for all to enjoy.

Ongoing evaluation The quick five question evaluation form centres around how the session has made our clients "feel" in terms of enjoyment, confidence, inner peace and energy. Results so far indicate significantly improved feelings and we have found clients eager to join the next group, often bringing in donations as well as their advice and expertise!

Conclusion We are hopeful that following final evaluations, results will indicate a positive impact on those who have attended sessions and will lead to further funding to continue HT in our day hospice setting. 